\title{
Chronic pulmonary aspergillosis in patients with bronchiectasis
}

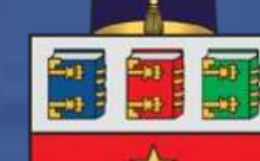

\author{
O. Munteanu, V. Botnaru, I. Volosciuc, E. Scutaru
}

State University of Medicine and Pharmacy "Nicolae Testemitanu"

Chisinau, Republic of Moldova

\section{Background}

Aspergillus-related diseases can complicate patients with already established bronchiectasis and lung architectural disruption, for example, an aspergilloma in a patient with post-tuberculosis bronchiectasis.

Furthermore, other coexistent diseases such as chronic obstructive pulmonary disease or bronchial asthma can increase the risk of Aspergillus-related disease but also independently can be associated with bronchiectasis.

\section{Aim}

To evaluate the prevalence, clinical and radiological characteristics of chronic pulmonary aspergillosis (CPA) in patients with bronchiectasis.

\section{Methods}

All patients referred to the Moldovan National Bronchiectasis Centre with bronchiectasis between Dec 2009 and Dec 2017 were screened for CPA by HRCT, culture results and serum antiAspergillus IgG.

\section{Results}

Of 334 referred patients with bronchiectasis, post-tuberculosis bronchiectasis identified in 88 cases (26\%). A total of 14 CPA patients, which included $10(71 \%)$ males, had a median age of $55.7 \pm 14$. Four patients had a history of treated pulmonary tuberculosis, but calcifications as a possible imaging marker of "spontaneous recovery" were identified in 12 patients (86\%).

Asthma was present from childhood in 2 cases that developed $A B P A$, one of them was treated for pulmonary tuberculosis (4 episodes). The median interval between ABPA and onset of CPA was 5 years. Other underlying lung conditions included chronic obstructive pulmonary disease $(n=6 ; 42 \%)$ and previous thoracic surgery $(n=1)$.

All patients presented with chronic pulmonary or systemic symptoms, such as purulent sputum (100\%), cough (100\%), haemoptysis ( $n=10,71 \%$ ) and weight loss ( $n=11,78 \%)$. The most common location of cavitary lesions (figure 1 ) was the right lung $(n=8,57 \%)$.

Death occurred in 2 patients (14\%).
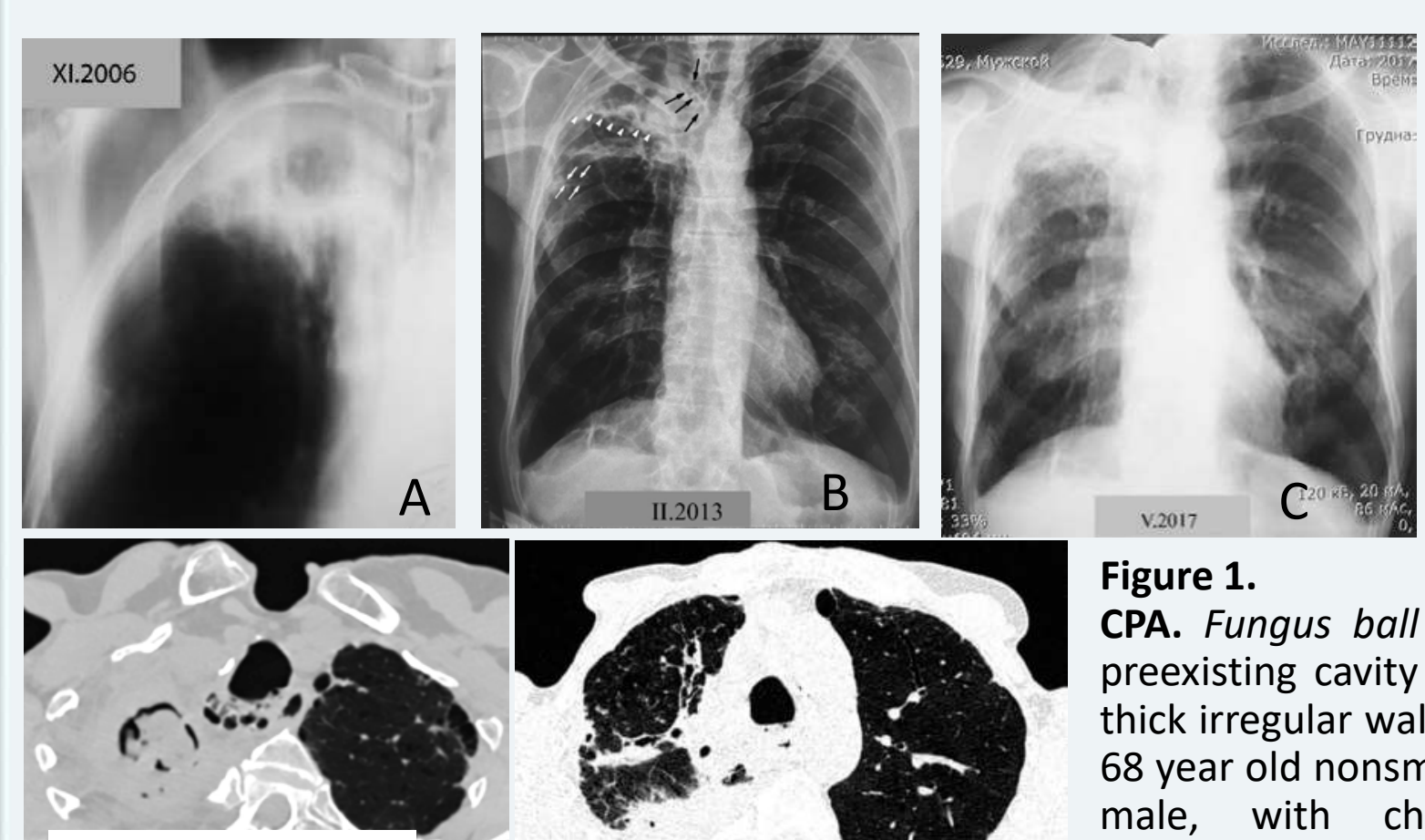

Figure 1.

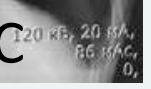

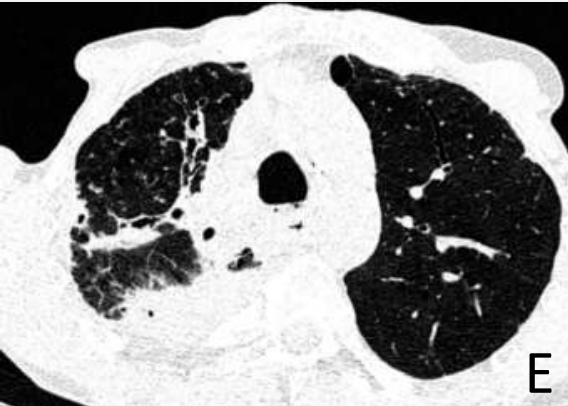

CPA. Fungus ball in a preexisting cavity with thick irregular wall in a 68 year old nonsmoker male, with chronic 29/05/2017 D E, cough. 11 years ago he

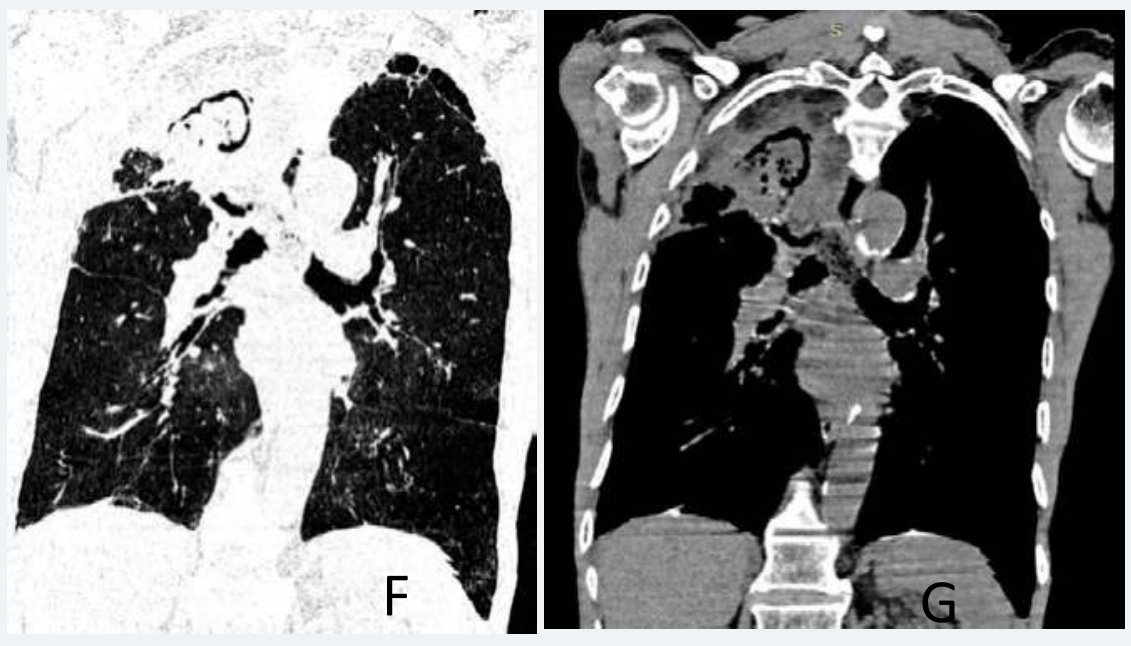
microbiologically negative pulmonary TB. Figure $1 \mathrm{~A}$ presents TB sequela at the completion of the TB treatment course. $\mathrm{He}$ was suspected for TB relapse in $05 / 2017$ according the radiological lesions(C) and progressive

worsening of his respiratory symptoms expressed by increasing amount of fou smelling purulent sputum (up to $50 \mathrm{ml} /$ day), progressive dyspnea and fatigue, weight loss $(\sim 8 \mathrm{~kg})$ and night sweats. Physical examination revealed pale skin, cachexia (IMC 15,8) and finger clubbing. HRCT revealed a huge cavity with fungus ball $(D, F, G)$, tracheocele $(D)$, cylindrical and varicose bronchiectasis $(E)$ and calcifications $(G)$.
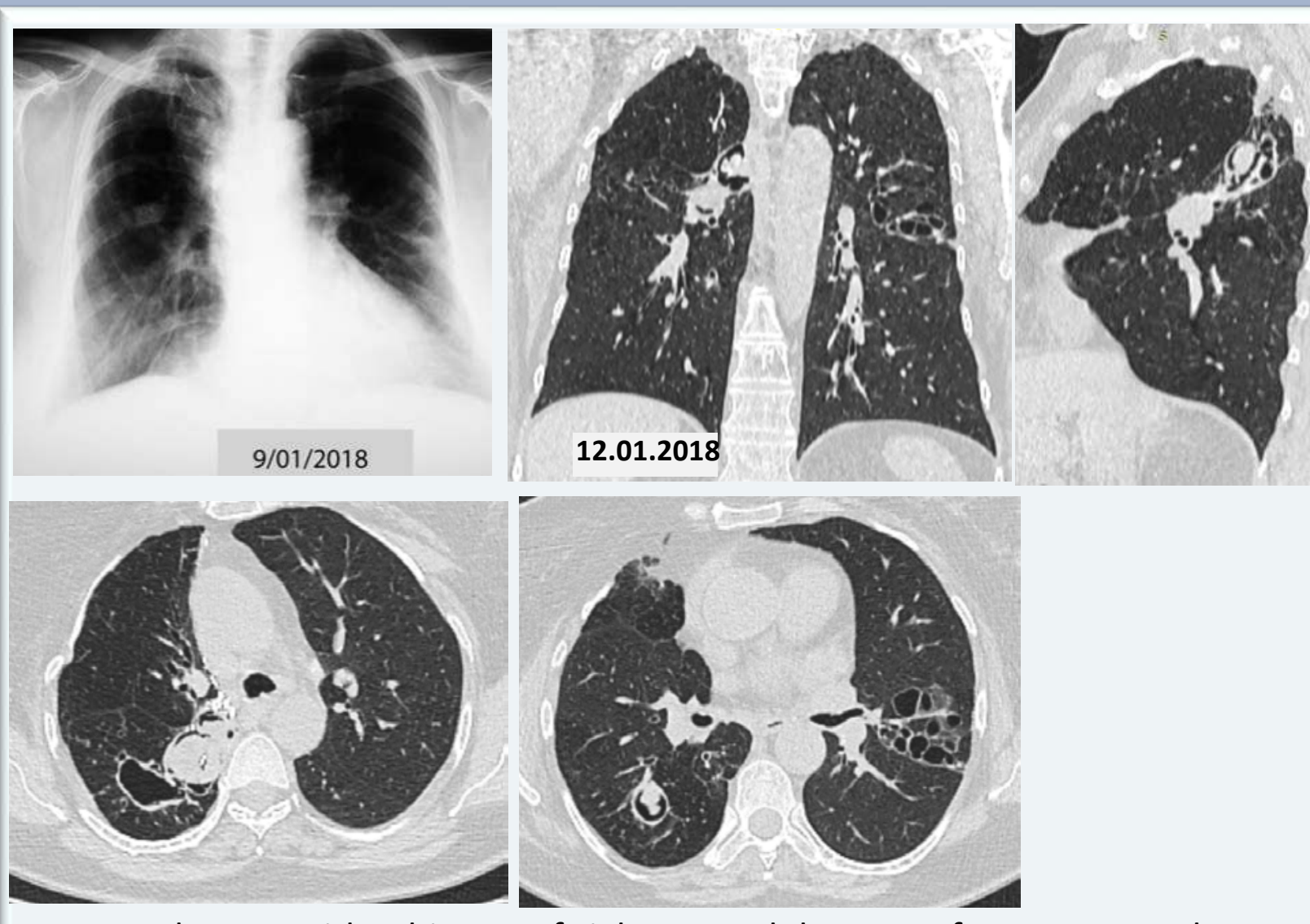

A woman known with a history of right upper lobectomy for trauma at the age of 15 was hospitalised with massive haemoptysis (up to $200 \mathrm{ml} /$ day). She was known with recurrent lower respiratory tract infections and chronic cough from childhood. HRCT images performed at the age of 61 show bilateral predominantly varicose and cystic bronchiectasis in the RML and lingula with multiple aspergillomas inside the cystic bronchiectasis.

\section{Conclusion}

Post-tuberculosis bronchiectasis is a common sequela in a high burden TB country and coexistent CPA may arise and should be considered, especially in smear negative patients suspected for TB reactivation. 